\title{
Negative Regulation of Nitric Oxide Release
}

National Cancer Institute

\section{Source}

National Cancer Institute. Negative Regulation of Nitric Oxide Release. NCI Thesaurus.

Code C41529.

Any process in which the release of the gaseous signaling molecule nitric oxide is reduced. Primarily in phagocytes, nitric oxide production is inhibited by transforming growth factor-beta, interleukin- 4 and interleukin-10. This process is involved in vasodilation, vessel homeostasis, immune response, neurotransmission and modulation of the hair cycle. 